\title{
The Increased Trend of Medical Treatment for Thyroid Diseases during Pregnancy: A 13-Year National Study
}

\author{
Suvi Turunen ${ }^{a}$ Marja Vääräsmäki ${ }^{a}$ Maarit Leinonen ${ }^{b}$ Mika Gissler ${ }^{b, c}$ \\ Tuija Männistö ${ }^{d}$ Eila Suvanto ${ }^{a}$ \\ aDepartment of Obstetrics and Gynecology, PEDEGO Research Unit, Medical Research Center Oulu, University of \\ Oulu and University Hospital of Oulu, Oulu, Finland; 'Information Services Department, Finnish Institute of Health \\ and Welfare, Helsinki, Finland; 'Department of Neurobiology, Care Sciences and Society, Karolinska Institute, \\ Stockholm, Sweden; dNorthern Finland Laboratory Centre Nordlab, Oulu, Department of Neurobiology, \\ Care Sciences and Society, Finland Karolinska Institute, Stockholm, Sweden
}

\section{Keywords}

Hypothyroidism · Hyperthyroidism • Pregnancy · Thyroid hormones · Antithyroid drugs

\begin{abstract}
Objective: Thyroid dysfunction affects up to $5-7 \%$ of all pregnancies. The rates of thyroid hormone use in nonpregnant population have substantially increased in recent years. The aim of this study was to assess possible changes in the use of levothyroxine substitution and antithyroid drugs over time in pregnant women. Methods: The study data consisted of all singleton pregnancies $(N=736,873$ ) between 2004 and 2016 in Finland collected from the Finnish Medical Birth Register. The Prescription Register and Special Refund Entitlement Register provided information on levothyroxine and antithyroid drug purchases. The annual rates of levothyroxine and antithyroid drug prescription redemptions were explored to estimate changes in exposure rates to thyroid medication from 2004 to 2016. Joinpoint regression analyses were performed to explore interannual variability in levothyroxine and antithyroid drug treatment. Results: There was more than a five-fold increase in levothyroxine use during the study period; in $2004,1.1 \%$ of pregnant women had le-
\end{abstract}

vothyroxine treatment, and by 2016, the prevalence increased to $6.2 \%$. In addition, we observed a slight increase in antithyroid medication during pregnancy, but antithyroid drug use during pregnancy overall was very rare. In 2004, $0.05 \%$ of pregnant women used antithyroid drugs, and by 2016, this percentage had increased to $0.14 \%$. Conclusions: Our study shows that the rate of levothyroxine use in pregnancy has markedly increased. This suggests that tracing and screening relevant patients and awareness of thyroid disorders on pregnancy and their significance for the pregnancy outcome have increased and the threshold to treat thyroid disorders has declined.

(c) 2021 European Thyroid Association Published by S. Karger AG, Basel

\section{Introduction}

Thyroid dysfunction affects up to $5-7 \%$ of all pregnancies [1]. Hypothyroidism in pregnant women is common with a prevalence of about $2-3 \%$, and the prevalence of undiagnosed subclinical hypothyroidism in pregnancy is 3-15\% [2]. Hyperthyroidism during pregnancy occurs in $0.4-1.7 \%$ of pregnant women $[3,4]$. Both overt hypo- and hyperthyroidism as well as subclinical hypothyroidism
Correspondence to:

Suvi Turunen, suvi.t.turunen@gmail.com 
and thyroid autoimmunity have been associated with adverse obstetrical and neonatal events such as preeclampsia and preterm birth $[2,5]$. Untreated thyroid disorders may threaten fertility and pregnancy [2] as well as harm the physical and mental development of the fetus $[6,7]$.

It is important to treat thyroid disorders during pregnancy since the potential benefits outweigh the potential harms [2]. Current American and European guidelines recommend treating overt maternal hypothyroidism with levothyroxine (LT4) and overt hyperthyroidism with antithyroid drugs (ATDs) including propylthiouracil and methimazole and its prodrug carbimazole $[3,8,9]$. Finland's national guidelines for treating thyroid disorders during pregnancy align with international guidelines [10]. It is acknowledged that overt thyroid diseases need to be treated, but there has been debate about whether or not to treat subclinical thyroid disorders. The 2017 guidelines of the American Thyroid Association stated that administration of LT4 to thyroid peroxidase antibody (TPO ab)-positive euthyroid pregnant women may be considered in some cases [3], but a recent study showed that this treatment had no effect on pregnancy or neonatal outcomes in women with a history of reproductive failure [11]. Additionally, subclinical hyperthyroidism has not been associated with adverse pregnancy outcomes [12], and thus ATDs, which may also have serious side effects [2], are not recommended in these cases.

The rates of thyroid hormone prescriptions in the general population have substantially increased in the USA and the UK [13] as well as in Finland in recent years [14]. In addition, the costs of managing thyroid diseases have increased continuously, mainly due to the rising costs of thyroid hormone therapy [15]. The increase of thyroid medication may, at least to some extent, be due to the lowering of the thyrotropin threshold for treatment [13, 16], increased awareness of thyroid diseases, or increased screening for thyroid disorders. Since thyroid hormone use in nonpregnant population has increased, we wanted to explore the use of LT4 and ATDs during pregnancy in Finland and to assess changes in these rates between 2004 and 2016.

\section{Methods}

The data used in this study were collected from four Finnish nationwide registers and include all singleton pregnancies $(734,773$ live births and 2100 stillbirths; $N=736,873$ ) between 2004 and 2016. This study period was chosen based on data availability. Our data were obtained from the Finnish Medical Birth Register (MBR), which was linked with the Special Refund Entitlement
Register, the Prescription Register, and the Care Register for Health Care (HILMO). These registers are of good quality $[17,18]$, and combining them increases their validity [19]. Linkage of the data from MBR and the other registers was performed using the unique personal identification codes that are assigned to all Finnish citizens and permanent residents. All personal identification codes were encrypted before analysis.

Information on maternal thyroid diseases was collected from the MBR, the Special Refund Entitlement Register, and the HILMO. The MBR was established in 1987, and it is maintained by the Finnish Institute for Health and Welfare. A structured form for the MBR is completed within 7 days after delivery by personnel at the delivery hospitals. This form includes maternal and newborn data for all live births and stillbirths with a gestational age at birth of $\geq 22$ weeks or birth weight of $\geq 500$ g. The Special Refund Entitlement Register is maintained by the Social Insurance Institution of Finland, Kela, and it includes information on chronic diseases as well as medication and reimbursement of medical expenses. The HILMO includes information on diagnoses from all hospital wards at discharge and specialized outpatient visits. The data from the HILMO spanned the years 1987-2016, while those from the Special Refund Entitlement Register ranged from 2004 to 2016.

We used the codes of the International Statistical Classification of Diseases and Related Health Problems (ICD) to identify hypothyroid (ICD-8 or ICD-9 code 244 or ICD-10 code E03 with all digits) and hyperthyroid (ICD-8 or ICD-9 code 242 or ICD-10 code E05 with all digits) women. In this study, women were classified as hypothyroid if they had a diagnosis for hypothyroidism in any of the registers before or during the pregnancy and/or they had levothyroxine medication 3 months before or during the pregnancy. Accordingly, they were classified as hyperthyroid if they had a recorded diagnosis for hyperthyroidism before or during the pregnancy and/or antithyroid drug medication 3 months before or during the pregnancy. In addition, women who had ICD-10 code O99.2 (endocrine, nutritional, and metabolic diseases complicating pregnancy, childbirth, and puerperium) recorded during pregnancy and documented LT4 purchases (see below) were classified as hypothyroid and those with ICD-10 code O99.2 and ATD purchases were classified as hyperthyroid. In Finland, hypothyroidism is mostly diagnosed and treated at primary health care also during pregnancy. In contrast, hyperthyroidism is diagnosed and managed at tertiary care. Nearly all women in Finland give birth at public hospitals (99.7\% during the study period), and diagnoses of their chronic diseases are recorded by attending midwives.

Data on thyroid medication purchases 3 months prior to and during pregnancy were obtained from the Statistics on Reimbursement for Medical Expenses, which includes the Special Refund Entitlement Register and the Prescription Register and is maintained by Kela. These registers collect information from all Finnish pharmacies on all prescription-only medication purchases as well as information related to the medicine (the International Anatomic Therapeutic Chemical classification code and the time and number of purchases). In our study, use of thyroid medication was defined as redeeming a prescription for LT4 (codes H03A01-05), propylthiouracil (code H03BA02), or carbimazole (code $\mathrm{H} 03 \mathrm{BB} 01$ ). The prescriptions of methimazole were also explored, but no methimazole usage was observed during the study years. Our data did not include information on LT4 or ATD treatment duration or dosage. 
Fig. 1. Pregnancies with maternal hypothyroidism and/or LT4 medication by year. LT4, levothyroxine.

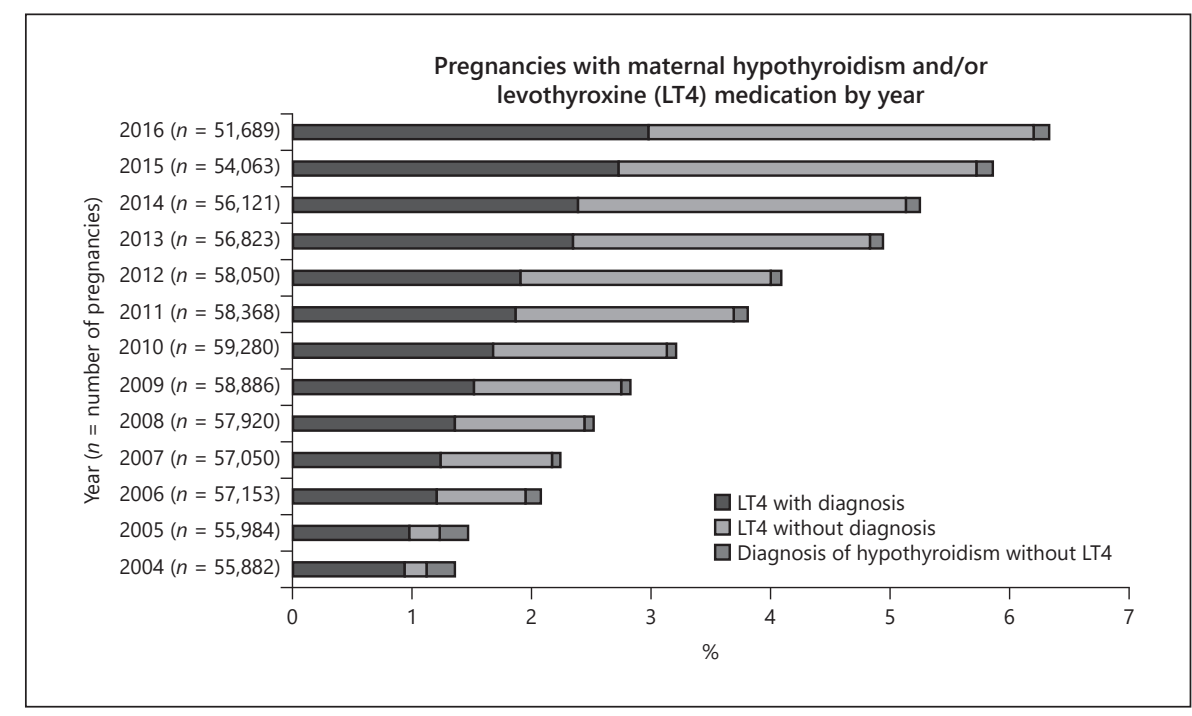

\section{Outcomes}

The hypothyroid women were classified as exposed or not exposed to LT4, and the hyperthyroid women were classified as exposed or not exposed to any ATD medication during pregnancy. We explored the annual rates of LT4 and ATD prescription redemptions to estimate changes in the rates of thyroid medication among pregnant women between 2004 and 2016.

\section{Statistical Analyses}

Oracle SQL Developer and SAS Enterprise Guide 7.1 were used to manage data. Joinpoint regression analyses were performed to examine trends over time. Joinpoint regression software uses permutation tests to identify points where linear trends change significantly. Joinpoint regression calculates an annual percentage change for each time period identified (1 year in this study). Joinpoint Regression Program Version 4.7.0.0 was used for the analyses.

\section{Results}

\section{Demographic Data}

Of all pregnancies $(N=736,873)$ between 2004 and 2016 in Finland, there were LT4 purchases in 24,953 (3.38\%) and ATD purchases in $714(0.10 \%)$. The demographic characteristics of mothers changed gradually between 2004 and 2016; mothers who gave birth in 2016 were older and more likely to be overweight or obese than mothers who gave birth in 2004 ( $p<0.001)$ (supporting information is given in online suppl. Table 1).

\section{LT4 Treatment}

The rates of LT4 use from 2004 to 2016 are presented in Figure 1 (supporting information is given in online suppl. Table 2). There was more than a five-fold increase in

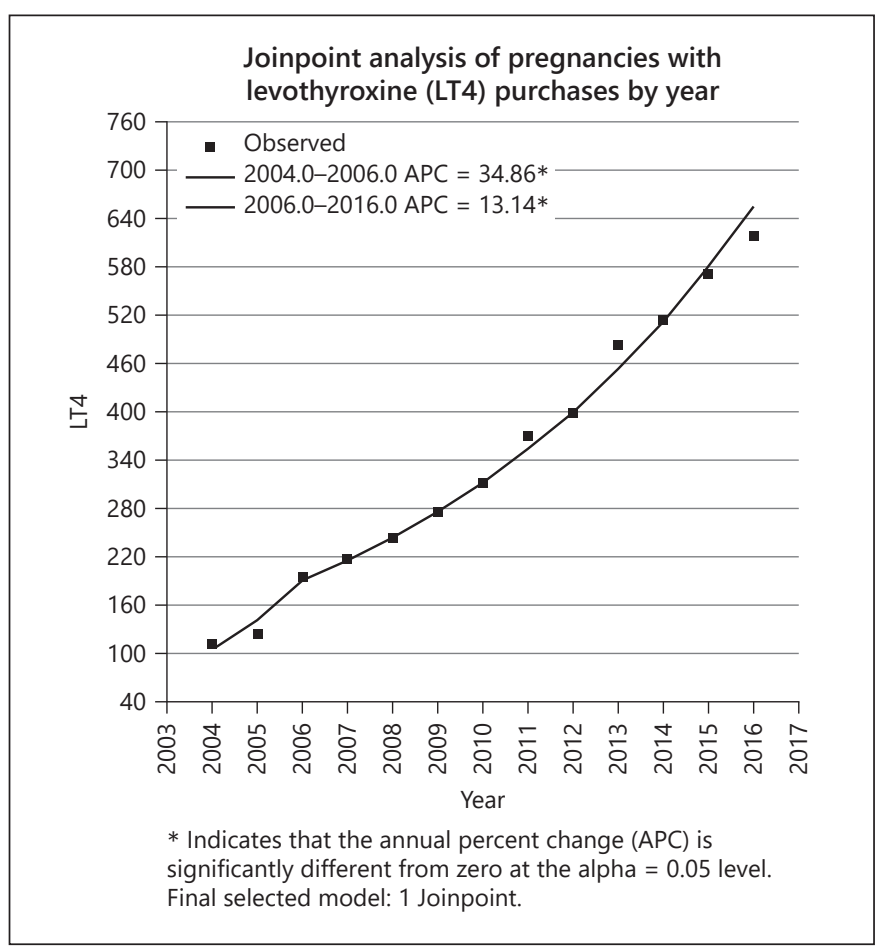

Fig. 2. Joinpoint analyses of pregnancies with LT4 medication by year. LT4, levothyroxine; APC, annual percent change.

LT4 use during the study period; in 2004, 1.1\% of pregnant women had LT4 treatment, and by 2016, the prevalence increased to $6.2 \%$. The prevalence of diagnosed hypothyroidism in the registers was $1.2 \%$ in 2004 and $3.1 \%$ in 2016. A portion of pregnant women received LT4 treatment 
Fig. 3. Pregnancies with maternal hyperthyroidism and/or ATD medication by year. ATD, antithyroid drug.

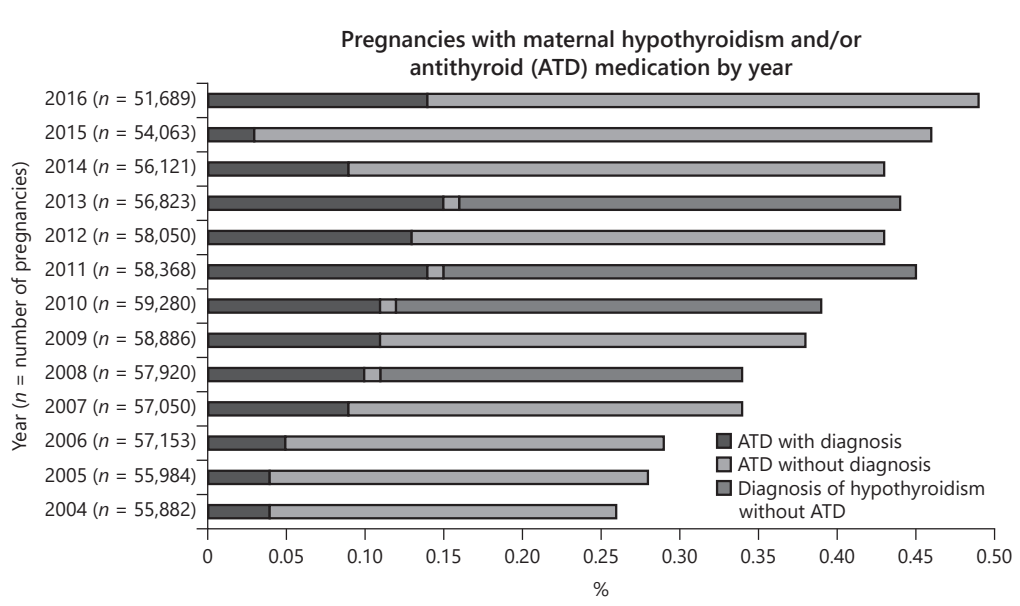

without a recorded hypothyroidism diagnosis $(3.2 \%$ in 2016). The vast majority of women with hypothyroidism purchased LT4 3 months before pregnancy or during the first trimester in both 2004 and 2016 (83.3 and 88.2\%, respectively; supporting information is given in online suppl. Table 3). The joinpoint regression analysis revealed the highest annual percent change (34.9\%) between 2004 and 2006. Otherwise, the increase in levothyroxine purchases among pregnant women has been linear over time (Fig. 2).

\section{ATD Treatment}

Use of ATDs also slightly increased during the study period, as presented in Figure 3 (supporting information is given in online suppl. Table 4). The number of pregnant women with hyperthyroid disease who were treated with ATDs increased from 0.05 to $0.14 \%$ during this time. Carbimazole was the most commonly purchased ATD $(N=$ 687 ), with only 37 propylthiouracil purchases during the whole study period. In 10 pregnancies, mothers with hyperthyroidism purchased both ATDs. The prevalence of hyperthyroidism slightly increased from $0.26 \%$ in 2004 to $0.49 \%$ in 2016 . In most cases, ATD refills occurred 3 months before pregnancy or in the first trimester (supporting information is given in online suppl. Table 3). The joinpoint regression analysis of ATD medication between 2004 and 2016 revealed no statistically significant changes (Fig. 4).

\section{Discussion}

In this study, we found more than a five-fold increase (from 1.12 to $6.19 \%$ ) in LT4 use among pregnant women during the study period (2004-2016). However, the prev-

\footnotetext{
The Trend of Thyroid Medication during
} Pregnancy

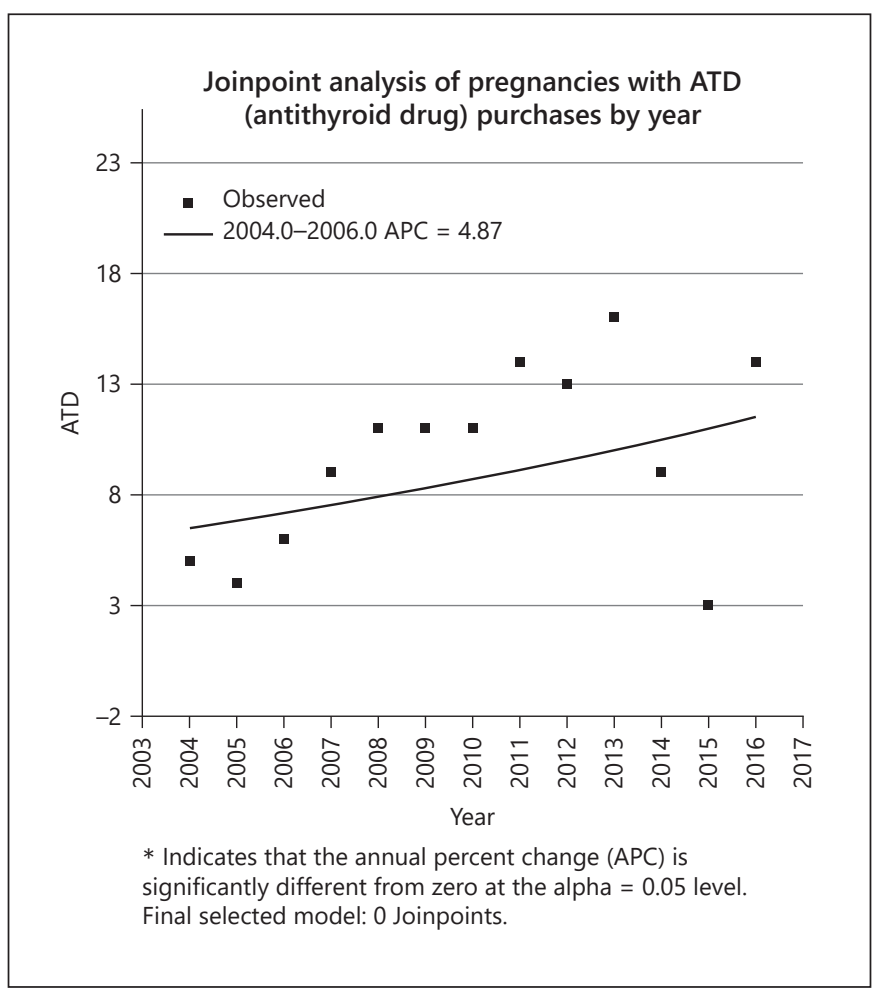

Fig. 4. Joinpoint analyses of pregnancies with ATD medication by year. ATD, antithyroid drug; APC, annual percent change.

alence of diagnosed hypothyroidism did not increase to the same extent. At the end of the study period, half of the pregnant women who used levothyroxine had no recorded diagnosis of hypothyroidism as determined by ICD codes. Also, the prevalence of diagnosed hyperthyroid- 
ism and the use of ATDs increased from 0.26 to $0.49 \%$ and 0.05 to $0.14 \%$, respectively, during this period.

There are only a few comparable studies on LT4 use during pregnancy. A Danish study showed an increase in LT4 use among pregnant women between 1997 and 2010 [20]. However, the authors reported that $<1 \%$ of pregnant women received LT4 medication, and this proportion is significantly lower than the estimated rate of hypothyroidism in pregnant women (about 2.8\%) [20]. Studies on nonpregnant populations have reported a substantial increase in the rate of thyroid hormone prescriptions [13]. Also, in the general population, the treatment costs of hypothyroidism have increased in recent years, but the costs for ATD have not substantially increased [15]. Our study showed a slight increase in the ATD redemption rate during pregnancy. In recent studies from Sweden and Denmark, 0.065 and $0.19 \%$ of pregnant women received ATD medication [21, 22], consistent with our results. However, there are no comparable studies on changes in exposure rates over time. In our study, carbimazole was the treatment of choice in the majority of pregnancies complicated with maternal hyperthyroidism, and propylthiouracil use was very rare. This finding is in contrast to international guidelines that recommend propylthiouracil use in the first trimester. Plausible explanation for the low propylthiouracil use is that propylthiouracil has been associated with severe hepatotoxicity and it can be prescribed only with special permission. Therefore, local and national treatment practices in Finland have favored using carbimazole.

The proportion of pregnant women exposed to LT4 was very high in the last years of our study. The joinpoint analysis showed a linear increase in LT4 purchases among pregnant women during the study period with one exception: the highest annual percent change (34.9\%) occurred between 2004 and 2006. There is no national screening program to diagnose thyroid disease in pregnant women in Finland. However, tracing and screening of relevant patients (e.g., a history of hyper- or hypothyroidism, symptoms related to thyroid disease, or a familial disposition to thyroid disease) is included in local recommendations given at maternity health clinics. In 2008, the Finnish Endocrinology Society stated that the dose of LT4 should be increased by $25 \mu \mathrm{g}$ as soon as the pregnancy test is positive [23]. Moreover, it was recommended that LT4 be started if the level of thyroid-stimulating hormone (TSH) in blood exceeded 2.5-3.0 mU/L [23]. The updated Finnish national guidelines published in 2014 had no relevant changes [10]. The introduction of the national guidelines may have increased the rate of prescription redemptions.
Studies addressing the risks associated with maternal thyroid disorders have been published before and during the study period $[2,6,7,24]$. Increased awareness of the importance of treatment may have prompted more frequent use of thyroid medication during pregnancy. There might also have been changes in screening and treatment practices during the study period. In our study, the prevalence of diagnosed maternal hypothyroidism has increased, and thus diagnostic activity has undoubtedly increased. Increased testing of thyroid hormones in nonpregnant population has also occurred, so more patients with subclinical disease are found [25]. In addition, obesity and maternal age at delivery have increased during the study period, both of which have been associated with thyroid disorders [26], and this might partly explain the increase in the prevalence of thyroid disorders. However, the proportion of LT4 users without a recorded diagnosis of hypothyroidism has also increased, which may demonstrate that the indication for treatment is lower than previously reported. However, it is very likely that the register on ICD codes is biased by underreporting due to general practitioners initiating LT4 treatment rather than tertiary care. Moreover, pregnant women with LT4 medication with no recorded diagnosis of hypothyroidism as determined by ICD codes may actually have a subclinical hypothyroidism or a diagnosis of hypothyroidism as determined by pregnancy thresholds which do not fulfill the criteria of clinical hypothyroidism.

Some guidelines have recommended LT4 treatment for patients with subclinical hypothyroidism at least in more severe forms $[9,27]$. Consequently, the proportion of general patients with subclinical hypothyroidism that have been treated with LT4 has increased [13]. International guidelines recommend that treatment with LT4 should be started in pregnancy in cases of subclinical hypothyroidism with thyroid autoantibodies with a TSH greater than the pregnancy-specific reference range and also in women without thyroid autoantibodies if TSH is $>10.0 \mathrm{mU} / \mathrm{L}$ [3]. It is very likely that there are mothers with medically treated subclinical hypothyroidism in our study cohort as well. Therefore, it should be noted that some authors have recently criticized current liberal use of LT4 treatment in cases with subclinical hypothyroidism [28]. The low TSH threshold for the treatment of subclinical hypothyroidism may lead to medicalizing women and overtreatment despite a lack of clear evidence that LT4 improves pregnancy outcome. In addition, careful consideration of potential risks and benefits of LT4 treatment during pregnancy is crucial because some recent studies have demonstrated that LT4 treatment of subclin- 
ical hypothyroidism may associate with adverse pregnancy outcomes [29] or even adverse neurodevelopment of the child [30].

\section{Strengths and Limitations}

We used information from nationwide registers that cover practically all births in Finland. There is no risk of recall bias since the information is collected from the registers rather than by questionnaires or interviews. However, since the MBR data are based on births, women with early pregnancy loss have not been included. Another limitation of our study was that we had no information about adherence to medication, only information on redeemed prescriptions. In addition, there were no laboratory data to confirm the thyroid hormone or iodine status of the mothers. However, according to traditional guidelines, blood sampling and laboratory testing are mandatory before medical treatment, and it is advised that thyroid hormone concentrations are followed regularly during treatment. Iodine insufficiency should not confound our study as Finland has been one of the few countries with sufficient iodine intake [31].

\section{Conclusion}

The use of LT4 among pregnant women has markedly increased during the past 13 years in Finland, and the use of ATDs in hyperthyroid women has increased slightly. This suggests that the awareness of thyroid disorders on pregnancy and also their significance for the pregnancy outcome has increased, and tracing and screening of relevant patients has improved. It also appears that the threshold to treat hypothyroidism and hyperthyroidism during pregnancy seems to be declined.

\section{Acknowledgement}

The authors thank Dr. Tapani Ebeling (Oulu University Hospital, Department of Endocrinology) for his assistance with the design of the research.

\section{Statement of Ethics}

The ethical board of the Northern Ostrobothnia Hospital District approved this study on October 13, 2014 (EETTMK: 80/2014). TheFinnishInstituteforHealthandWelfare(THL/928/5.05.00/2014 and THL/408/5.05.00/2019) and Kela (Kela 75/522/2014 and Kela $25 / 522 / 2019)$ gave permission to access the data from the aforementioned national health registers. The Northern Ostrobothnia District partly funded this study. The data were encrypted prior to the statistical analysis. This is a register-based study in which the study subjects were not contacted.

\section{Conflict of Interest Statement}

Prof. Gissler and Dr. Leinonen report that they received grants from the Innovative Medicines Initiative (Building an ecosystem for better monitoring and communicating the safety of medicines' use in pregnancy and breastfeeding: validated and regulatory endorsed workflows for fast, optimized evidence generation, IMI ConcePTION, Grant Agreement No. 821520) while conducting the study. The other authors (Dr. Turunen, Dr. Vääräsmäki, Dr. Männistö, and Dr. Suvanto) have explicitly stated that there are no conflicts of interest related to this article.

\section{Funding Sources}

This work was supported in part by the Northern Ostrobothnia Hospital District (Dr. Turunen), the Drugs and Pregnancy Project of the National Institute for Health and Welfare (THL), Finnish Medicines Agency (FIMEA), and Social Insurance Institution of Finland (Kela) (Prof. Gissler and Dr. Leinonen).

\section{Author Contributions}

All authors contributed to the conception and design of the research and to the acquisition, analysis, and interpretation of the data. Dr. Turunen drafted the initial version of the manuscript, and other authors revised it critically for important intellectual content and approved the final version to be published. All authors agree to be accountable for all aspects of the work and to ensure that questions related to the accuracy or integrity of any part of the work are appropriately investigated and resolved. Dr. Turunen attests that all listed authors meet the authorship criteria and that no others meeting the criteria have been omitted.

\section{References}

The Trend of Thyroid Medication during Pregnancy
1 Dong AC, Stagnaro-Green A. Differences in diagnostic criteria mask the true prevalence of thyroid disease in pregnancy: a systematic review and meta-analysis. Thyroid. 2019 Feb; 29(2):278-89.

2 Negro R, Stagnaro-Green A. Clinical aspects of hyperthyroidism, hypothyroidism, and thyroid screening in pregnancy. Endocr Pract. 2014 Jun;20(6):597-607.
3 Alexander EK, Pearce EN, Brent GA, Brown RS, Chen H, Dosiou C, et al. 2017 guidelines of the american thyroid association for the diagnosis and management of thyroid disease during pregnancy and the postpartum. Thyroid. 2017 Mar;27(3):315-89.

4 Cooper DS, Laurberg P. Hyperthyroidism in pregnancy. Lancet Diabetes Endocrinol. 2013 Nov;1(3):238-49. 
5 Negro R, Stagnaro-Green A. Diagnosis and management of subclinical hypothyroidism in pregnancy. BMJ. 2014 Oct6;349:g4929.

6 Moog NK, Entringer S, Heim C, Wadhwa PD, Kathmann N, Buss C. Influence of maternal thyroid hormones during gestation on fetal brain development. Neuroscience. 2017 Feb7; 342:68-100.

7 Pop VJ, Brouwers EP, Vader HL, Vulsma T, van Baar AL, de Vijlder JJ. Maternal hypothyroxinaemia during early pregnancy and subsequent child development: a 3-year followup study. Clin Endocrinol. 2003 Sep;59(3): 282-8.

8 De Groot L, Abalovich M, Alexander EK, Amino N, Barbour L, Cobin RH, et al. Management of thyroid dysfunction during pregnancy and postpartum: an Endocrine Society clinical practice guideline. J Clin Endocrinol Metab. 2012 Aug;97(8):2543-65.

9 Pearce SH, Brabant G, Duntas LH, Monzani F, Peeters RP, Razvi S, et al. ETA guideline: management of subclinical hypothyroidism. Eur Thyroid J. 20132013 Dec;2(4):215-28.

10 Suomen Endokrinologiyhdistyksen suositus kilpirauhasen sekä lisämunuaiskuoren vajaatoiminnan diagnostiikasta ja hoidosta [Internet]. Available from. https://www.endo.fi/@ Bin/179635/Hypotyreoosilausunto_051114_ final.pdf.

11 Dhillon-Smith RK, Middleton LJ, Sunner KK, Cheed V, Baker K, Farrell-Carver S, et al. Levothyroxine in women with thyroid peroxidase antibodies before conception. N Engl J Med. 2019 Apr 4;380(14):1316-25.

12 Casey BM, Leveno KJ. Thyroid disease in pregnancy. Obstet Gynecol. 2006 Nov; 108(5): 1283-92.

13 Taylor PN, Iqbal A, Minassian C, Sayers A, Draman MS, Greenwood R, et al. Falling threshold for treatment of borderline elevated thyrotropin levels-balancing benefits and risks: evidence from a large communitybased study. JAMA Intern Med. 2014 Jan; 174(1):32-9.

14 Soininen M. Joka kolmas tyroksiinihoito voi olla turha. Suomen Lääkärilehti. 2017;72:407.

15 Mollehave LT, Linneberg A, Skaaby T, Knudsen N, Ehlers L, Jørgensen T, et al. Trends in costs of thyroid disease treatment in Denmark during 1995-2015. Eur Thyroid J. 2018 Mar;7(2):75-83.

16 Medici BB, Nygaard B, la Cour JL, Grand MK, Siersma V, Nicolaisdottir DR, et al. Changes in prescription routines for treating hypothyroidism between 2001 and 2015: an observational study of 929,684 primary care patients in copenhagen. Thyroid. 2019 Jul;29(7):910-9.

17 Teperi J. Multi method approach to the assessment of data quality in the Finnish Medical Birth Registry. J Epidemiol Community Health. 1993 Jun;47(3):242-7.

18 Sund R. Quality of the finnish hospital discharge register: a systematic review. Scand J Public Health. 2012 Aug;40(6):505-15.

19 Gissler M, Louhiala P, Hemminki E. Nordic medical birth registers in epidemiological research. Eur J Epidemiol. 1997 Feb;13(2):16975.

20 Gidén K, Andersen JT, Torp-Pedersen AL, Enghusen Poulsen H, Torp-Pedersen C, Jimenez-Solem E. Use of thyroid hormones in relation to pregnancy: a Danish nationwide cohort study. Acta Obstet Gynecol Scand. 2015 Jun;94(6):591-7.

21 Andersen SL, Lönn S, Vestergaard P, Törring $O$. Birth defects after use of antithyroid drugs in early pregnancy: a Swedish nationwide study. Eur J Endocrinol. 2017 Oct;177(4): 369-78.

22 Schurmann L, Hansen AV, Garne E. Pregnancy outcomes after fetal exposure to antithyroid medications or levothyroxine. Early Hum Dev. 2016 Oct;101:73-7.
23 Schalin-Jäntti C. Kilpirauhassairaudet ja raskaus. Suom Lääkäril. 2008;35:2795-800.

24 Hou J, Yu P, Zhu H, Pan H, Li N, Yang H, et al. The impact of maternal hypothyroidism during pregnancy on neonatal outcomes: a systematic review and meta-analysis. Gynecol Endocrinol. 2016;32(1):9-13.

25 Bayram C, Valenti L, Britt H. Orders for thyroid function tests-changes over 10 years. Aust Fam Physician. 2012 Aug;41(8):555.

26 Turunen S, Vääräsmäki M, Männistö T, Hartikainen AL, Lahesmaa-Korpinen AM, Gissler M, et al. Pregnancy and perinatal outcome among hypothyroid mothers: a population-based cohort study. Thyroid. 2019 Jan; 29(1):135-41.

27 Surks MI, Ortiz E, Daniels GH, Sawin CT, Col $\mathrm{NF}$, Cobin RH, et al. Subclinical thyroid disease: scientific review and guidelines for diagnosis and management. JAMA. 2004 Jan 14; 291(2):228-38.

28 Wiles KS, Jarvis S, Nelson-Piercy C. Are we overtreating subclinical hypothyroidism in pregnancy? BMJ. 2015;351:h4726.

29 Maraka S, Mwangi R, McCoy RG, Yao X, Sangaralingham LR, Singh Ospina NM, et al. Thyroid hormone treatment among pregnant women with subclinical hypothyroidism: US national assessment. BMJ. 2017 Jan 25;356: i6865.

30 Korevaar TI, Muetzel R, Medici M, Chaker L, Jaddoe VW, de Rijke YB, et al. Association of maternal thyroid function during early pregnancy with offspring IQ and brain morphology in childhood: a population-based prospective cohort study. Lancet Diabetes Endocrinol. 2016 Jan;4(1):35-43.

31 Nyström HF, Brantsæter AL, Erlund I, Gunnarsdottir I, Hulthén L, Laurberg P, et al. Iodine status in the Nordic countries - past and present. Food Nutr Res. 2016 Jun 8;60: 31969. 